\title{
Fat- and fiber-related diet behavior among type 2 diabetes patients from distinct regions
}

This article was published in the following Dove Press journal:

Patient Preference and Adherence

19 February 2015

Number of times this article has been viewed

\section{Tereza Hendrychova' Magda Vytrisalova' \\ Abdullah Alwarafi ${ }^{2}$ Jurjen Duintjer Tebbens ${ }^{3,4}$ Helena Vankatova' Sandra Leal ${ }^{5}$ Ales Antonin Kubena' Alena Smahelova ${ }^{6}$ Jiri Vlcek'}

'Department of Social and Clinical Pharmacy, Faculty of Pharmacy in Hradec Králové, Charles University in Prague, Hradec Králové, Czech Republic; ${ }^{2}$ Faculty of Dentistry, lbb University, Ibb, Yemen; ${ }^{3}$ Department of Biophysics and Physical Chemics, Faculty of Pharmacy in Hradec Králové, Charles University in Prague, Hradec Králové, Czech Republic; ${ }^{4}$ Institute of Computer Science, Academy of Sciences of the Czech Republic, Prague, Czech Republic; ${ }^{5}$ Department of Clinical Pharmacy, El Rio Community Health Center, Tucson, AZ, USA; ${ }^{6}$ Diabetes Center, Department of Gerontology and Metabolism, University Hospital and Faculty of Medicine in Hradec Králové, Charles University in Prague, Hradec Králové, Czech Republic
Correspondence: Magda Vytrisalova Department of Social and Clinical Pharmacy, Faculty of Pharmacy in Hradec Králové, Charles University in Prague, Heyrovského I203, 50005 Hradec Králové, Czech Republic

Tel +420 49506727 I

Fax +420 495512266

Email magda.vytrisalova@faf.cuni.cz
Purpose: Diet and eating habits are of key importance in patients with type 2 diabetes mellitus (T2DM). The purpose of this comparative study was to analyze fat- and fiber-related behavior (FFB) in patients with T2DM from distinct cultural areas.

Patients and methods: Observational study was carried out in the Czech Republic (CR) $(n=200)$, the US $(n=207)$, and Yemen $(n=200)$. Patients completed the Fat- and Fiber-related Diet Behavior Questionnaire (FFBQ).

Results: Differences in all aspects of FFB among countries were found $(P<0.05)$. The best fat-related behavior reported was from patients from the CR. Patients from the US showed the worst fat-related behavior in total. On the other hand, patients from the US reported the best fiber-related behavior. Patients from Yemen reached the worst scores in all fat-related domains. Patients from all studied countries reported the best results in the "modify meat" and "avoid fat as flavoring" and the worst in the "substitute high fiber" subscales.

Conclusion: Professionals involved in the diet education of T2DM patients should be aware of the specificity of diet in their country when advising patients keeping general recommendations. We suggest them to be as specific as possible and concentrate on fiber-related behavior.

Keywords: type 2 diabetes mellitus, fat-related behavior, fiber-related behavior, Fat- and Fiberrelated Diet Behavior Questionnaire, geographical difference

\section{Introduction}

Diabetes mellitus is one of the most common chronic diseases in both the developed and developing countries. ${ }^{1}$ Worldwide prevalence in 2000 was estimated to be 171 million, and this is projected to increase to at least 366 million by the year $2030 .^{2}$

Changing lifestyles characterized by reduced physical activity, sedentary behavior, and unhealthy dietary habits are linked with type 2 diabetes mellitus (T2DM). ${ }^{3}$ Optimal treatment of this metabolic disease requires comprehensive management, of which diet is a critical part. The key aspects of a diabetic diet include reduction of saturated fat intake and an incremental increase of dietary fiber intake. ${ }^{4-6}$ It has been recently confirmed that dietary fiber improves postprandial glycemia in diabetes due to slowing of gastric emptying. ${ }^{7}$

It has been shown that adults with T2DM make different food choices than nondiabetic adults, which can result in higher saturated fat and lower fiber intake. ${ }^{8}$

The guidelines concerning diet recommendations for T2DM patients are patterned on the American Diabetes Association (ADA) advice all over the world. According to a Spanish study, ${ }^{9}$ over one-half of adult diabetic patients do not follow these recommendations. As one of the reasons, we suppose that such universal recommendations can be insufficient. However, it is known that for the best possible adherence of a patient to the recommended diet, it is necessary to provide personalized education with 
emphasis on the current eating habits taking into account his or her preferences and the culture ${ }^{4}$ this could be inadequately kept in practice. Culture and cultural habits are key factors of food intake, ${ }^{10}$ so it is useful to examine the differences in diet among various countries.

Up to now, the work published on dietary habits, dealing with fat-related behavior in particular, ${ }^{11-15}$ has been mainly from the US. A broader spectrum of information on the dietary habits in a few European countries was provided by a study by Boylan et al. ${ }^{16}$ However, to our knowledge, this has been inadequately studied in patients with T2DM.

Data from the Czech Republic $(\mathrm{CR})^{17}$ concerning this group of patients have shown healthier fat-related behavior than fiber-related one. Patients modify the meals rather than remove them completely from their diet or replace them by other types of food. In our further research, we aimed at analyzing and comparing the eating habits regarding fat- and fiber-related behavior (FFB) in patients with T2DM from distinct regions.

We hypothesize that this research may point out relevant differences in particular areas of FFB among diverse populations. This information may make the work of health care providers involved in dietary education of T2DM patients from various parts of the world more effective. This could increase the adherence of patients with dietary recommendations, which is of utmost importance in the comprehensive management of T2DM.

\section{Methods Study design}

This cross-sectional questionnaire study was carried out in three countries: the CR (the University Hospital in Hradec Králové and two diabetes outpatient clinics within the region) from March to June 2011; the US (the Pharmacy-Based Diabetes Program in Tucson, Arizona) from September to November 2011, and in Yemen (the Nasser Hospital in Ibb) from February to March 2013. The study protocol was reviewed and approved by the regional Ethics Committee.

\section{Study population}

Participants were recruited directly at the study places on randomly selected days. Consecutive patients who agreed to complete the questionnaire and met the enrollment criteria were surveyed. The enrollment criteria were as follows: patients had a diagnosis of T2DM for at least 3 months or more from the date of contact, and the patient's ability to understand the questionnaire. All participants provided informed consent.

\section{Instruments and measures}

The study subjects completed the Fat- and Fiber-related Diet Behavior Questionnaire (FFBQ), ${ }^{18}$ which has been described in detail elsewhere. ${ }^{17}$ There are four response alternatives to each question (always, often, sometimes, never). Higher scores indicate unsound diet behavior, ie, higher fat and lower fiber intake. Summary scale for fat intake, summary scale for fiber intake, and eight subscales (five for fat and three for fiber intake) were calculated as proposed by the originators of the instrument. The tool had been translated into Czech for the CR and Arabic for Yemen. In the US, a modified English version and Spanish version were used. Each language version of the questionnaire was adapted to typical local dietary particularities by means of appropriate wording when necessary (eg, adjusted terms for types of available bakery products) in a particular country. The questionnaire, containing also information on age, gender, and age at T2DM diagnosis, was either self-administered or investigator-assisted.

Forward-backward translation by two independent translators and review by a native speaker were carried out for each language version. Based on piloting among ten patients with T2DM in each country, no modifications were then required. Cronbach's $\alpha$ values of particular language versions of the questionnaire are stated in Table 1.

Type of diabetes treatment, drugs used to control diabetes, measurements for glycosylated hemoglobin (A1C), waist circumference, and body height and weight were obtained from medical records. Body mass index was calculated based on the most recent height and weight measurements. When a particular value was missing, it was measured in situ by the competent person. A1C was measured according to the

Table I Cronbach's $\alpha$ values of particular language versions of the questionnaire

\begin{tabular}{lll}
\hline $\begin{array}{l}\text { Language } \\
\text { version }\end{array}$ & Part of the & $\begin{array}{l}\text { Cronbach's } \\
\alpha \text { value }\end{array}$ \\
\hline Czech & questionnaire & 0.772 \\
& Dietary fat intake (24 items) & 0.484 \\
English & Dietary fiber intake (I3 items) & 0.749 \\
& Dietary fat intake (24 items) & 0.466 \\
Spanish & Dietary fiber intake (I3 items) & 0.635 \\
& Dietary fat intake (24 items) & 0.509 \\
Arabic & Dietary fiber intake (I3 items) & 0.662 \\
& Dietary fat intake (24 items) & 0.461 \\
\hline
\end{tabular}


Table 2 Basic characteristics of the study cohort

\begin{tabular}{|c|c|c|c|c|c|}
\hline Characteristic & $\begin{array}{l}\text { Full cohort ( } n=607 \\
49.1 \% \text { men) }\end{array}$ & $\begin{array}{l}\text { Czech Republic } \\
\text { ( } n=200 ; 54.5 \% \text { men) }\end{array}$ & $\begin{array}{l}\text { US ( } n=207 \\
38.2 \% \text { men) }\end{array}$ & $\begin{array}{l}\text { Yemen ( } n=200 ; \\
55 \% \text { men) }\end{array}$ & $\begin{array}{l}P \text {-value (Kruskal- } \\
\text { Wallis test) }\end{array}$ \\
\hline Age (years) & $57.6 \pm 13.2$ & $66.2 \pm 10.1$ & $56.8 \pm 12.6$ & $50.0 \pm 11.3$ & $<0.00$ I \\
\hline Age at diabetes diagnosis (years) & $48.2 \pm 12.6$ & $54.4 \pm 10.9$ & $45.3 \pm 12.7$ & $45.1 \pm 11.7$ & $<0.001$ \\
\hline BMI $\left(\mathrm{kg} / \mathrm{m}^{2}\right)$ & $30.6 \pm 6.4$ & $31.1 \pm 5.0$ & $33.3 \pm 7.8$ & $27.4 \pm 4.1$ & $<0.00$ I \\
\hline Waist circumference $(\mathrm{cm})$ & $105.0 \pm 16.5$ & $104.6 \pm 11.2$ & $110.6 \pm 18.2$ & $99.6 \pm 16.9$ & $<0.001$ \\
\hline DCCT AIC (\%) & $7.7 \pm 1.9$ & $7.6 \pm 1.7$ & $8.0 \pm 1.9$ & $7.6 \pm 2.0$ & 0.006 \\
\hline
\end{tabular}

Notes: Data are mean \pm SD. a Multi-ethnic population was involved: $18.4 \%$ of Caucasian, $24.6 \%$ of Native Americans, $52.2 \%$ of Hispanics, and $4.8 \%$ of Asians. Abbreviations: SD, standard deviation; BMI, body mass index; DCCT, Diabetes Control and Complications Trial; AIC, glycated hemoglobin.

standard procedure and paid for by the investigator, as it is not commonly monitored in diabetic patients in Yemen.

\section{Data analysis}

The outcome of the FFB questionnaire is summarized using means and standard deviations by item and by the eight subscales and the two overall summary scores (for FFB). Items are further characterized with percentages. To test whether the country of residence has an influence on the answers to individual items, the $P$-values of Pearson's $\chi^{2}$ test for homogeneity and Fisher's exact test or of the $\chi^{2}$ test after rebinning were computed. When testing whether the country of residence has an influence on the subscales and main scales, $\chi^{2}$ and one-way Kruskal-Wallis tests were performed. The PASW statistical software was used for all analyses (IBM Corporation, Armonk, NY, USA, v 18.0), except for Fisher's exact tests, which were performed with the online calculator on the webpage http://www.physics.csbsju.edu/ stats/exact NROW NCOLUMN form.html.

\section{Results}

\section{Study population}

Data from 200 patients in the CR, 207 patients from the US, and 200 patients from Yemen were analyzed. The basic characteristics of the study cohort are given in Table 2. Table 3 shows the characteristics of diabetes treatment in the study cohort.

\section{Fat-related behavior}

Findings regarding FFB in the studied countries are shown in Table 4. There were significant differences in fat-related diet habits summary scale scores and in all fat-related behavior subscales scores among countries. The only one exception was the "replace fruits and vegetables" subscale scores for the $\mathrm{CR}$ and the US. Overall, the best fat-related behavior reported was from diabetic patients from the CR. Patients from the US showed the worst fat-related behavior in total. Patients from all studied countries reported the best results in the "modify meat" and "avoid fat as flavoring" subscales coincidentally.

\section{Fiber-related behavior}

Diabetic patients from Yemen scored significantly worse than those from the CR and the US in fiber-related diet habits summary scale. Patients from the US reported the best fiber-related diet behavior in all fiber-related subscales, while diabetic patients from Yemen reached the worst scores in all cases. Patients from the CR and the US showed no differences in substituting of high-fiber foods for low-fiber variants. They both scored the worst in this area. Patients from Yemen reported the worst results in the "cereals and grains" subscale score.

\section{Timing of meals}

Diabetic patients in the US stated eating breakfast and lunch less often in comparison with the two other countries

Table 3 Characteristics of diabetes treatment in the study cohort

\begin{tabular}{|c|c|c|c|c|c|}
\hline Type of diabetes treatment* & $\begin{array}{l}\text { Full cohort }(n=607 \text {; } \\
49.1 \% \text { men })\end{array}$ & $\begin{array}{l}\text { Czech Republic } \\
\text { ( } n=200 ; 54.5 \% \text { men) }\end{array}$ & $\begin{array}{l}\text { US ( } n=207, \\
38.2 \% \text { men })\end{array}$ & $\begin{array}{l}\text { Yemen }(n=200 ; \\
55 \% \text { men })\end{array}$ & $\begin{array}{l}\text { P-value } \\
\text { (Kruskal-Wallis test) }\end{array}$ \\
\hline Diet alone & 8.4 & 5.1 & 9.7 & 10.5 & $<0.001$ \\
\hline Oral antidiabetic drugs & 62.0 & 63.6 & 48.8 & 74.0 & $<0.001$ \\
\hline Insulin therapy & 15.0 & 16.2 & 14.0 & 15.0 & $<0.001$ \\
\hline Oral antidiabetic drugs + insulin therapy & 14.5 & 15.2 & 27.5 & 0.5 & $<0.001$ \\
\hline Mean number of antidiabetic drugs ${ }^{a}$ & $\mathrm{I} .54$ & 1.7 & $\mathrm{I} .83$ & 1.14 & $<0.001$ \\
\hline
\end{tabular}

Notes: Data are percentage. *Statistically significant difference between countries $\left(P<0.000 \mathrm{I}\right.$ according to the Kruskal-Wallis test). ${ }^{\mathrm{a}} \mathrm{For}$ combination drugs, each active ingredient is taken into account separately, as is each type of insulin used. 
Table 4 Items and scales of the Fat- and Fiber-related Diet Behavior Questionnaire

\begin{tabular}{|c|c|c|c|c|c|c|c|c|}
\hline \multirow{2}{*}{$\begin{array}{l}\text { How often did you... } \\
\text { Item/scale }\end{array}$} & \multicolumn{3}{|l|}{ Mean \pm SD } & \multicolumn{3}{|c|}{$\begin{array}{l}\text { Percentage of subjects } \\
\text { reporting scores } I \text { and } 2\end{array}$} & \multicolumn{2}{|l|}{$P$-value } \\
\hline & Czech Republic & US & Yemen & Czech Republic & US & Yemen & Pearson $\chi^{2}$ & Other tests \\
\hline Eat broiled, baked, or poached fish? & $2.4 I \pm 1.00$ & $2.3 \pm 1.02$ & $2.45 \pm 1.18$ & 50.6 & 55.2 & 47.0 & 0.078 & \\
\hline Eat broiled or baked chicken? & $1.97 \pm 0.77$ & $1.99 \pm 0.86$ & $\mathrm{I} . \mathrm{I} \pm 0.40$ & 74.1 & 69.3 & 97.5 & $<0.000 \mathrm{I}^{\mathrm{r}}$ & \\
\hline Take the skin off chicken? & $2.8 \mathrm{I} \pm 1.30$ & $1.83 \pm 1.15$ & $1.88 \pm 1.28$ & 37.5 & 71.3 & 69.8 & $<0.0001$ & \\
\hline Eat pasta or noodles without meat? & $2.57 \pm 0.80$ & $2.8 \pm 1.00$ & $2.79 \pm 1.25$ & 46.6 & 33.5 & 33.0 & $<0.0001$ & \\
\hline Eat whole-wheat pasta or noodles? & $3.5 \mathrm{I} \pm 0.73$ & $3.4 I \pm 0.93$ & $4.00 \pm 0.00$ & 10.9 & 14.9 & 0.0 & $<0.000$ I & \\
\hline Trim visible fat from red meat? & $2.00 \pm 1.18$ & $2.04 \pm 1.13$ & $1.86 \pm 1.16$ & 64.8 & 62.9 & 74.6 & 0.035 & \\
\hline Eat extra lean ground meat? & $2.59 \pm 1.14$ & $2.12 \pm 1.08$ & $3.94 \pm 0.4 I$ & 49.4 & 59.8 & 1.9 & $<0.0001$ & \\
\hline $\begin{array}{l}\text { Eat bread, rolls, or crackers without } \\
\text { butter or margarine? }\end{array}$ & $2.58 \pm 0.96$ & $2.37 \pm 1.08$ & $2.82 \pm 1.24$ & 43.1 & 51.4 & 37.4 & $<0.0001$ & \\
\hline $\begin{array}{l}\text { Eat whole grain types of bread, } \\
\text { rolls, or crackers? }\end{array}$ & $2.47 \pm 1.01$ & $2.11 \pm 1.14$ & $3.98 \pm 0.22$ & 47.5 & 61.1 & 0.5 & $<0.0001$ & \\
\hline Eat high-fiber cereal or add dried fruit? & $2.87 \pm 1.04$ & $2.36 \pm 0.96$ & $4.00 \pm 0.00$ & 34.2 & 55.0 & 0.0 & 0.016 & $0.014^{e}$ \\
\hline $\begin{array}{l}\text { Add bran or some type of fiber to } \\
\text { cereal? }\end{array}$ & $3.22 \pm 1.02$ & $3.27 \pm 0.94$ & $4.00 \pm 0.00$ & 25.0 & 19.2 & 0.0 & 0.91 & $0.84^{\mathrm{e}}$ \\
\hline Use low-fat or nonfat milk? & $2.55 \pm 1.26$ & $2.63 \pm 1.34$ & $3.55 \pm 0.98$ & 48.9 & 44.0 & 13.6 & $<0.000$ I & \\
\hline Eat specially made low-fat cheese? & $2.43 \pm 1.04$ & $3.24 \pm 1.10$ & $4.0 \pm 0.00$ & 50.3 & 21.9 & 0.0 & $<0.000$ I & \\
\hline Eat low-fat or nonfat frozen dessert? & $3.10 \pm 0.96$ & $2.94 \pm 1.00$ & $3.68 \pm 0.70$ & 23.1 & 28.6 & 7.9 & 0.001 & \\
\hline $\begin{array}{l}\text { Add butter, margarine, or other } \\
\text { fat to cooked vegetables? }\end{array}$ & $2.30 \pm 1.08$ & $2.27 \pm 1.16$ & $2.69 \pm 1.26$ & 34.8 & 40.0 & 57.5 & $<0.0001$ & \\
\hline Eat fried vegetables? ${ }^{\text {a }}$ & $1.53 \pm 0.66$ & $1.38 \pm 0.76$ & $1.02 \pm 0.22$ & 5.7 & 8.1 & 0.5 & $<0.000 \mathrm{I}^{\mathrm{r}}$ & \\
\hline Eat fried potatoes? ${ }^{\mathrm{a}}$ & $1.56 \pm 0.58$ & $2.21 \pm 0.94$ & $1.63 \pm 1.03$ & 3.6 & 33.9 & 17.6 & $<0.000 \mathrm{I}$ & \\
\hline $\begin{array}{l}\text { Add butter, margarine, or sour } \\
\text { cream to potatoes? }\end{array}$ & $2.47 \pm 1.08$ & $2.93 \pm 1.04$ & $1.00 \pm 0.00$ & 51.5 & 27.7 & 100.0 & $<0.0001$ & \\
\hline Eat brown rice? & $3.54 \pm 0.68$ & $3.32 \pm 0.97$ & $4 \pm 0$ & 7.4 & 17.3 & 0.0 & $<0.0001$ & \\
\hline Eat salads without dressing? & $2.40 \pm 1.19$ & $2.95 \pm 1.12$ & $2.46 \pm I .46$ & 51.9 & 29.5 & 50.8 & $<0.0001$ & \\
\hline $\begin{array}{l}\text { Eat salads with low-fat or nonfat } \\
\text { dressing? }\end{array}$ & $2.57 \pm 1.20$ & $2.69 \pm 1.25$ & $4.00 \pm 0.00$ & 48.4 & 39.3 & 0.0 & $<0.0001$ & \\
\hline $\begin{array}{l}\text { Eat no meat, fish, eggs, or cheese at } \\
\text { dinner? }\end{array}$ & $2.99 \pm 0.85$ & $3.23 \pm 0.80$ & $2.95 \pm 1.16$ & 24.7 & 15.5 & 33.0 & $<0.0001$ & \\
\hline Eat two or more vegetables at dinner? & $2.77 \pm 0.77$ & $2.60 \pm 0.92$ & $1.70 \pm 1.04$ & 39.1 & 42.0 & 79.0 & $<0.0001$ & \\
\hline Eat one or more vegetables at lunch? & $2.49 \pm 0.77$ & $2.54 \pm 0.96$ & $2.99 \pm 0.84$ & 45.8 & 45.9 & 19.7 & $<0.0001$ & \\
\hline Eat fresh fruit at breakfast? & $3.57 \pm 0.65$ & $2.75 \pm 0.99$ & $3.9 \pm 0.40$ & 7.9 & 36.3 & 2.6 & $<0.000 \mathrm{I}$ & \\
\hline Eat cereal or oats at breakfast? & $3.79 \pm 0.52$ & $2.51 \pm 1.06$ & $3.97 \pm 0.30$ & 4.2 & 48.0 & 1.0 & $<0.0001$ & \\
\hline $\begin{array}{l}\text { Add cream or whipped cream } \\
\text { to dessert? }\end{array}$ & $1.45 \pm 0.82$ & $1.63 \pm 0.82$ & $1.32 \pm 0.80$ & 9.9 & 12.9 & 8.5 & $3.00 E-4^{r}$ & \\
\hline Eat only fruit for dessert? & $2.70 \pm 0.89$ & $2.61 \pm 0.93$ & $3.45 \pm 0.90$ & 38.5 & 40.2 & 13.3 & $<0.0001$ & \\
\hline Eat raw vegetables as a snack? & $2.85 \pm 0.85$ & $2.93 \pm 0.83$ & $3.58 \pm 0.67$ & 36.4 & 25.9 & 9.9 & $<0.0001$ & \\
\hline Eat fresh fruit as a snack? & $2.49 \pm 0.81$ & $2.44 \pm 0.9$ & $3.46 \pm 0.67$ & 48.7 & 47.1 & 9.9 & $<0.000 \mathrm{I}$ & \\
\hline Use olive oil when fry? & $2.58 \pm 1.13$ & $2.78 \pm 1.17$ & $3.58 \pm 0.82$ & 48.7 & 33.6 & 12.5 & $<0.0001$ & \\
\hline $\begin{array}{l}\text { Trim visible fat from red meat } \\
\text { before cooking? }\end{array}$ & $2.02 \pm 1.19$ & $2.06 \pm 1.17$ & $1.83 \pm 1.13$ & 65.3 & 62.1 & 76.0 & 0.083 & \\
\hline Eat low-fat or nonfat mayonnaise? & $2.90 \pm 1.09$ & $3.09 \pm 1.08$ & $3.63 \pm 0.74$ & 32.7 & 25.0 & 12.5 & 0.315 & $0.355^{\mathrm{e}}$ \\
\hline $\begin{array}{l}\text { Use less fat when bake cookies } \\
\text { or cakes? }\end{array}$ & $2.75 \pm 1.16$ & $3.18 \pm 0.9$ & $3.61 \pm 0.86$ & 35.4 & 20.3 & 13.2 & $<0.000$ I & \\
\hline Subscales* & & & & & & & & \\
\hline Modify meat & $2.30 \pm 0.69$ & $2.05 \pm 0.67$ & $1.82 \pm 0.67$ & & & & $<0.000 \mathrm{I}^{\mathrm{r}}$ & $<0.000 \mathrm{I}^{\mathrm{k}}$ \\
\hline Avoid fat as flavoring & $2.09 \pm 0.49$ & $2.24 \pm 0.56$ & $1.91 \pm 0.54$ & & & & $<0.000 \mathrm{I}^{\mathrm{r}}$ & $<0.000 \mathrm{I}^{\mathrm{K}}$ \\
\hline Replace, meat & $2.79 \pm 0.58$ & $3.07 \pm 0.74$ & $2.97 \pm 1.01$ & & & & $<0.000 \mathrm{I}^{\mathrm{r}}$ & $<0.000 \mathrm{I}^{\mathrm{K}}$ \\
\hline Substitute low fat & $2.6 \mathrm{I} \pm 0.73$ & $2.89 \pm 0.76$ & $3.85 \pm 0.34$ & & & & $<0.000 \mathrm{I}^{\mathrm{r}}$ & $<0.000 \mathrm{I}^{\mathrm{K}}$ \\
\hline Replace, fruits and vegetables & $2.7 I \pm 0.67$ & $2.66 \pm 0.72$ & $3.47 \pm 0.7 \mid$ & & & & $<0.000 \mathrm{I}^{\mathrm{r}}$ & $<0.000 \mathrm{I}^{\mathrm{K}}$ \\
\hline Fruits and vegetables & $2.86 \pm 0.49$ & $2.64 \pm 0.60$ & $2.98 \pm 0.47$ & & & & $<0.000 \mathrm{I}^{\mathrm{r}}$ & $<0.000 \mathrm{I}^{\mathrm{K}}$ \\
\hline Substitute high fiber & $3.34 \pm 0.48$ & $3.32 \pm 0.63$ & $3.56 \pm 0.56$ & & & & $0.004^{r}$ & $<0.000 \mathrm{I}^{\mathrm{K}}$ \\
\hline
\end{tabular}


Table 4 (Continued)

\begin{tabular}{|c|c|c|c|c|c|c|c|c|}
\hline \multirow{2}{*}{$\begin{array}{l}\text { How often did you... } \\
\text { Item/scale }\end{array}$} & \multicolumn{3}{|l|}{ Mean \pm SD } & \multicolumn{3}{|c|}{$\begin{array}{l}\text { Percentage of subjects } \\
\text { reporting scores I and } 2\end{array}$} & \multicolumn{2}{|l|}{$P$-value } \\
\hline & Czech Republic & US & Yemen & Czech Republic & US & Yemen & Pearson $\chi^{2}$ & Other tests \\
\hline Cereals and grains & $3.16 \pm 0.61$ & $2.59 \pm 0.74$ & $3.98 \pm 0.18$ & & & & $<0.000 \mathrm{I}^{\mathrm{r}}$ & $<0.000 \mathrm{I}^{\mathrm{K}}$ \\
\hline \multicolumn{9}{|l|}{ Main scales* } \\
\hline Fat-related diet habits summary scale & $2.39 \pm 0.42$ & $2.46 \pm 0.4 I$ & $2.44 \pm 0.35$ & & & & $0.014^{r}$ & $0.115^{\mathrm{K}}$ \\
\hline $\begin{array}{l}\text { Fiber-related diet habits summary } \\
\text { scale }\end{array}$ & $3.06 \pm 0.39$ & $2.79 \pm 0.44$ & $3.39 \pm 0.29$ & & & & $<0.000 \mathrm{I}^{\mathrm{r}}$ & $<0.000 \mathrm{I}^{\mathrm{K}}$ \\
\hline
\end{tabular}

Notes: aReverse scoring (always - never =4-I). 'P-value after rebinning, the answers "always" and "usually" are associated with the same outcome. eP-value of Fisher's exact test. ${ }^{K} P$-value of Kruskal-Wallis test. *The percentage of subjects reporting scores I and 2 was not counted for the subscales and main scales.

Abbreviation: SD, standard deviation.

$(P<0.0001)$, and those in the $\mathrm{CR}$ indicated eating snacks more often than those in the two other countries $(P<0.0001)$.

\section{Discussion}

The present study was designed to compare the eating habits regarding FFB in patients with T2DM from distinct geographical and cultural areas and to point out relevant differences in this field. We chose one country each from Europe, America, and the Near East, which we think are geographically and culturally diverse enough to detect any relevant differences in the eating habits.

The results concerning the basic characteristics of the study cohorts from different countries are in accordance with data published, ${ }^{1,19}$ which show higher prevalence of diabetes in lower age groups (45-64 years) in developing countries and among people belonging to a racial or ethnic minority groups.

The FFBQ is a psychometrically valuable instrument, valid and reliable, ${ }^{16}$ which has been used in several studies. $^{20,21}$ In all language versions, lower values of the Cronbach's $\alpha$ concerning the fiber-related part are referred to the wider scope of items in this part.

Although various language versions of the questionnaire were used, they were the same in their meaning. Piloting of each version should further increase the quality of the instrument.

Despite the fact that all T2DM patients should be receiving advice on increasing fiber intake and decreasing fat intake, ${ }^{4-6}$ the results show that they vary in the extent to which they stick to this diet behavior. Based on our results, it is obvious that diabetic patients comply better with the fatrelated component of the recommended diet than fiber-related one. This is in accordance with the results of Beresford et $\mathrm{al}^{11}$ who evaluated the effectiveness of self-help materials for dietary intervention in a primary care. Mostly, diabetic patients from Yemen seem to have problems with sufficient intake of fiber. The most difficult part of fiber-related behavior in the CR and the US is, coincidentally, substitution of low-fiber foods for high-fiber ones, while in Yemen it seems to be the consumption of cereals and grains. Cereals are a conventional part of the diet in the US, which the presented results confirmed, whereas the lack of specifically modified foods rich in fiber may be the main reason for low consumption of fiber in Yemen.

When we focus on fat-related behavior, we may suggest that diabetic patients modify the dishes rather than omit them from their diet completely, which agrees with the findings of Quandt et al. ${ }^{22}$ The most frequently adopted diet behavior for decreasing intake of fat is modification of meat (eg, removing all visible fat from meat) and avoiding fat as flavoring (eg, not using fat when preparing vegetables or potatoes) identically across the studied countries. Spoon et $\mathrm{al}^{23}$ in their study pointed out an association between "avoid fat as flavoring" and "modify meats", which supports our results. Further, significant variations or different scores of the "avoid fat as flavoring" subscale have been found in studies on dietary interventions, ie, among various ethnicities. ${ }^{12,13,18}$

Patients from Yemen performed the substitution of highfat foods with their low-fat variants less often than patients from the CR and the US, where this behavior is quite common. We suggest that it is caused by the lack of specifically modified foods low in fat in Yemen.

Despite the fact that diabetic patients from Yemen reporting intake of fruits and vegetables close to intake of these kinds of foods in the CR and the US, they seem to fail in substituting desserts and snacks containing fat for fruits and vegetables. This may be caused by the fact that they eat snacks less often than the diabetic patients in the other studied countries.

Adherence to both FFB is a cornerstone of the diet recommendations for patients with T2DM. As the guidelines (including diet recommendations) for the care of T2DM 
patients are all patterned on the ADA guideline, we suppose very similar diet education of patients all over the world and the differences in particular fields of diet shown in our study may be assigned mainly to cultural and geographical differences that we just aimed to analyze.

The main limitations of our study are the facts that our samples are not based on populations across the involved countries and that the patients were not randomly selected. We chose the days of the data collection randomly, and all patients who appeared for an ordinary appointment in the particular study place within these days were addressed.

Both fat- and fiber-related outcomes are based on patients' self-reports. Despite the fact that the survey was anonymous, social desirability bias, ie, the tendency to report more favorable behavior in accordance with societal expectations, could limit the accuracy of reporting with a distortion of absolute numbers. However, the influence of the potential bias is expected to be similar in all studied countries, so the comparison among them is still valuable.

To ensure the response rate as much as possible, patients' incomes and material conditions were not examined. Nevertheless, most of participants were seniors with a lower socioeconomic status. In the US and Yemen, the clinics where the surveys were conducted were a Federally Qualified Health Center (FQHC) and a governmental hospital, respectively, which often times serves people of lower socioeconomic status, but that is not exclusively the case. However, culture probably affects dietary habits independent of material conditions. $^{10}$

\section{Conclusion}

We found significant differences in all aspects of FFB among studied countries. The best fat-related behavior reported was from diabetic patients from the CR. Patients from the US reported the best fiber-related behavior. Modifying and adjusting dishes is more common than omitting their particular components from the diet or replacing them by other types of food among patients across countries.

According to our experience, it is necessary to further increase the general awareness of diabetic patients of the appropriate diet and support their adherence to it, eg, by focusing on the most problem areas. Professionals involved in the diet education of T2DM patients should be aware of the specificity of diet in their country when advising patients on general recommendations. It may be useful to communicate the diet recommendation in more detail and with focus on typical meals for each area. We suggest concentration on fiber-related behavior in all T2DM patients.

\section{Acknowledgments}

This study was supported by Mobility Fund and research grant No SVV 260066 from Charles University in Prague, Czech Republic. The work of J Duintjer Tebbens was also supported by the grant GA13-06684S of the Grant Agency of the Czech Republic.

\section{Disclosure}

The authors report no conflicts of interest in this work.

\section{References}

1. Whiting DR, Guariguata L, Weil C, Shaw J. IDF diabetes atlas: global estimates of the prevalence of diabetes for 2011 and 2030. Diabetes Res Clin Pract. 2011;94:311-321.

2. Wild S, Roglic G, Green A, Sicree R, King H. Global prevalence of diabetes: estimates for the year 2000 and projections for 2030. Diabetes Care. 2004;5:1047-1053.

3. Al-Habori M, Al-Mamari M, Al-Meeri A. Type II diabetes mellitus and impaired glucose tolerance in Yemen: prevalence, associated metabolic changes and risk factors. Diabetes Res Clin Pract. 2004;65: 275-281.

4. Inzucchi SE, Bergenstal RM, Buse JB, et al; American Diabetes Association (ADA); European Association for the Study of Diabetes (EASD). Management of hyperglycaemia in type 2 diabetes: a patient-centered approach. Position statement of the American diabetes association (ADA) and the European association for the study of diabetes (EASD). Diabetologia. 2012;55:1577-1596.

5. American Diabetes Association. Nutrition recommendations for diabetes: a position statement of the American diabetes association. Diabetes Care. 2008;31(suppl 1):S61-S78.

6. American Diabetes Association. Standards of medical care in diabetes - 2012. Diabetes Care. 2012;35(suppl 1):S11-S63.

7. Yu K, Ke MY, Li WH, Zhang SQ, Fang XC. The impact of soluble dietary fibre on gastric emptying, postprandial blood glucose and insulin in patients with type 2 diabetes. Asia Pac J Clin Nutr. 2014;23(2): 210-218.

8. Breen C, Ryan M, McNulty B, Gibney MJ, Canavan R, O’Shea D. High saturated-fat and low-fibre intake: a comparative analysis of nutrient intake in individuals with and without type 2 diabetes. Nutr Diabetes. 2014;4:e104.

9. Muñoz-Pareja M, León-Muñoz LM, Guallar-Castillón P, et al. The diet of diabetic patients in Spain in 2008-2010: accordance with the main dietary recommendations - A cross-sectional study. PLoS One. 2012; 7:e39454

10. Shatenstein B, Ghadirian P. Influences on diet, health behaviours and their outcome in select ethnocultural and religious groups. Nutrition. 1998; 14(2):223-230.

11. Beresford SAA, Farmer EMZ, Feingold L, Graves KL, Summer SK, Baker RM. Evaluation of a self-help dietary intervention in a primary care setting. Am J Public Health. 1992;82:79-84.

12. Kristal AR, White E, Shattuck AL, et al. Long-term maintenance of a low-fat diet: durability of fat-related dietary habits in the women's health trial. J Am Diet Assoc. 1992;92:553-559.

13. Kristal AR, Shattuck AL, Patterson E. Differences in fat-related dietary patterns between black, Hispanic and white women: results from the women's health trial feasibility study in minority populations. Public Health Nutr. 1999;2:253-262.

14. Hart A, Tinker L, Bowen DJ, Longton G, Beresford SAA. Correlates of fat intake behaviors in participants in the eating for a healthy life study. J Am Diet Assoc. 2006;106:1605-1613.

15. Satia JA, Galanko JA. Comparison of three methods of measuring dietary fat consumption by African-American adults. $J$ Am Diet Assoc. 2007;107:782-791. 
16. Boylan S, Welch A, Pikhart H, et al. Dietary habits in three Central and Eastern European countries: the HAPIEE study. BMC Public Health. 2009;9:439.

17. Hendrychova T, Vytrisalova M, Vlcek J, Smahelova A, Kubena AA. An analysis of fat-related and fiber-related behaviour in men and women with type 2 diabetes mellitus: key findings for clinical practice. Patient Prefer Adherence. 2013;7:877-884.

18. Shannon J, Kristal AR, Curry SJ, Beresford SAA. Application of a behavioral approach to measuring dietary change: the fat- and fiberrelated diet behavior questionnaire. Cancer Epidemiol Biomarkers Prev. 1997;6:355-361.

19. Satia JA. Diet-related disparities: understanding the problem and accelerating solutions. J Am Diet Assoc. 2009;109(4):610-615.
20. Bowen DJ, Beresford SA, Vu T, et al. Baseline data and design for a randomized intervention study of dietary change in religious organizations. Prev Med. 2004;39:602-611.

21. Hart A, Tinker LF, Bowen DJ, Satia-Aboutia J, McLerran D. Is religious orientation associated with fat and fruit/vegetable intake? J Am Diet Assoc. 2004;104:1292-1296.

22. Quandt SA, Bell RA, Snively BM, Vitolins MZ, Wetmore-Arkader LK, Arcury TA. Dietary fat reduction behaviors among African American, American Indian, and white older adults with diabetes. J Nutr Elder. 2009; 28(2):143-157.

23. Spoon MP, Devereux PG, Benedict JA, et al. Usefulness of the food habits questionnaire in a worksite setting. J Nutr Educ Behav. 2002; $34: 268-272$

\section{Publish your work in this journal}

Patient Preference and Adherence is an international, peer-reviewed, open access journal that focuses on the growing importance of patient preference and adherence throughout the therapeutic continuum. Patient satisfaction, acceptability, quality of life, compliance, persistence and their role in developing new therapeutic modalities and compounds to optimize clinical outcomes for existing disease states are major areas of interest for the journal. This journal has been accepted for indexing on PubMed Central. The manuscript management system is completely online and includes a very quick and fair peer-review system, which is all easy to use. Visit http://www. dovepress.com/testimonials.php to read real quotes from published authors.

\footnotetext{
Submit your manuscript here: http://www.dovepress.com/patient-preference-and-adherence-journal
} 